\title{
BENEFÍCIOS DA FISIOTERAPIA AQUÁTICA EM IDOSOS COM OSTEOARTROSE DE JOELHO
}

\author{
Bruna dos Santos Modesto ${ }^{1}$ \\ Kauara Vilarinho Santana Vieira ${ }^{2}$
}

RESUMO: A osteoartrose é uma patologia reumática crônico-degenerativa em que ocorre o estresse celular e por consequência a degradação da matriz extracelular. A fisioterapia aquática é um recurso fisioterapêutico que vem sendo bastante utilizado na recuperação de pacientes com doenças como a osteoartrose, por possuir uma grande vantagem decorrente das propriedades físicas e dos efeitos fisiológicos da água, ela auxilia na diminuição da sustentação de peso nas articulações, músculos e ossos, progredindo no fortalecimento muscular dos pacientes, além de facilitar os movimentos e elevar o encorajamento emocional para o retorno das atividades no solo. O objetivo deste estudo é evidenciar os benefícios da fisioterapia aquática em idosos com osteoartrose de joelho, como alívio dos sintomas, contribuindo para uma melhor funcionalidade e qualidade de vida. Trata-se de uma revisão bibliográfica onde os artigos foram acessados por meio de bases de dados publicados entre 20II a 202I. Foi possível concluir através deste estudo que, a fisioterapia aquática é um importante recurso na reabilitação dos pacientes com osteoartrose, trazendo o alívio de dores, aumentando a capacidade funcional de articulações acometidas, retorno da independência nas atividades diárias, e uma melhor qualidade de vida.

Palavras-chave: Osteoartrose. Hidroterapia. Fisioterapia.

ABSTRACT: Osteoarthrosis is a chronic-degenerative rheumatic pathology in which cellular stress occurs and, consequently, the degradation of the extracellular matrix. Aquatic physiotherapy is a physiotherapeutic resource that has been widely used in the recovery of patients with diseases such as osteoarthrosis, as it has a great advantage due to the physical properties and physiological effects of water, it helps to reduce weight bearing in joints, muscles and bones, progressing in the muscle strengthening of patients, in addition to facilitating movement and increasing emotional encouragement for the return of activities on the ground. The aim of this study is to demonstrate the benefits of aquatic physical therapy in elderly people with knee osteoarthritis, as symptom relief,

\footnotetext{
${ }^{I}$ Graduanda do curso de Fisioterapia Pela instituição de ensino Faculdade UNIBRAS- Rio Verde

${ }^{2}$ Fisioterapeuta, especialista em Traumato-Ortopedia e Desportiva Professora na Faculdade UNIBRAS- Rio Verde.
} 
contributing to better functionality and quality of life. This is a literature review where the articles were accessed through databases published between 20II and 202. I. It was possible to conclude through this study that aquatic physical therapy is an important resource in the rehabilitation of patients with osteoarthritis, bringing relief from pain, increasing the functional capacity of affected joints, return of independence in daily activities, and a better quality of life.

Keywords: Osteoarthrosis. Hydrotherapy. Physiotherapy.

\section{INTRODUÇÃO}

A osteoartrose (OA) é uma patologia reumática crônica degenerativa em que ocorre o estresse celular e por consequência a degradação da matriz extracelular caracterizada pelo desgaste da cartilagem, remodelação óssea, inflamação das articulações, formação de osteófitos e perda da função articular (SANTOS; AMARAL, 2020). A degradação que ocorre na cartilagem ocorre de forma lenta e leva ao quadro sintomático sendo que os principais sintomas são dor, crepitação, rigidez pela manhã, redução da amplitude de movimento, atrofia muscular, sobrecarga ligamentar e perda da função (VIEIRA et al., 2016).

Dentre todas as articulações do corpo, que podem ser acometidas pela OA, o joelho é evidenciado como uma das mais afetadas. Essa patologia atinge 6\% das pessoas adultas com mais de 30 anos, taxa essa que aumenta para 10\%, quando relacionada a pessoas com mais de 60 anos (KAWANO et al., 2015).

A degradação da cartilagem articular ocorre de maneira lenta e progressiva, ocasionada pela insuficiência do condrócito em manter o equilíbrio entre renovação e degradação tecidual, prevalecendo à degradação. Com isso ocorrem fissuras na cartilagem articular e em sua estrutura, beneficiando a perda funcional da articulação, reduzindo a capacidade de absorção do impacto e da mobilidade articular podendo apresentar os seguintes sintomas, dor articular, rigidez matinal, crepitação, entre outros sintomas (UTH; TROFONOV, 2014).

A atuação da fisioterapia é de extrema importância para a osteoartrose de joelho tanto no tratamento quanto na prevenção de agravos evitando que ocorra o avanço de deformidades e perda da capacidade funcional da articulação. A fisioterapia vai atuar 
através da utilização de recursos para reduzir a dor, melhorar a amplitude de movimento e manter

a função articular, resultando na melhora da qualidade de vida do indivíduo (SANTOS; AMARAL, 2020).

A hidroterapia é um recurso fisioterapêutico que utiliza uma piscina de água quente, como um agente de resistência para a execução de atividades terapêuticas. Atualmente, é notável o crescente aumento da utilização desta terapia no Brasil. Esta técnica leva em consideração as propriedades físicas da água (densidade relativa e gravidade, empuxo, megacentro, pressão hidrostática, água em movimento fluxo laminar ou turbulento, refração, tensão superficial e temperatura), utilizadas de maneira eficaz para melhorar a qualidade de vida do paciente (SILVA; GAMA, 2017).

A fisioterapia aquática pode ser considerada como uma das principais intervenções terapêuticas no tratamento da OA. As propriedades físicas e fisiológicas da água possibilitam a realização de exercícios dificilmente executados em solo, e que, associados à maior amplitude de movimento e à temperatura elevada da água, aumentam a mobilidade articular, o controle muscular e a resistência, aliviando dores e acelerando o processo de recuperação funcional (MIOTTO et al., 2013).

O objetivo deste estudo culmina em evidenciar os benefícios da fisioterapia aquática em idosos com osteoartrose de joelho, na redução da dor, em promover ganho de capacidade funcional, e uma melhor qualidade de vida.

\section{MATERIAIS E MÉTODOS}

Este artigo trata-se de uma revisão bibliográfica. O estudo foi realizado por meio de pesquisa em capítulos de livros, artigos científicos e revistas especializadas que buscam os benefícios da fisioterapia aquática em idosos com osteoartrose de joelho. Os descritores selecionados para a pesquisa serão: osteoartrose, hidroterapia, fisioterapia aquática, tratamento e osteoartrite, apenas em português. Foram levantados artigos realizados 
durante 2011 a 202I, por meio das bases de dados, Scielo, Google Acadêmico, BVS Biblioteca Virtual em Saúde.

\section{FUNDAMENTAÇÃO TEÓRICA}

\section{Definição e fisiopatologia}

A osteoartrose é uma doença osteoarticular degenerativa que acomete a cartilagem hialina iniciando com a deterioração local, progredindo para a degeneração dessa cartilagem,

gerando hipertrofia e deformação do osso subcondral devido a inflamações secundarias da membrana sinovial (CECHETTI et al., 2012).

Com o passar do tempo e com o processo do envelhecimento, tais tecidos podem sofrer um desgaste desenvolvendo lesões ocasionando alterações e desequilíbrios nessa articulação (OLIVEIRA et al., 2013).

É a artropatia mais comum e pode levar a grande incapacidade, principalmente quando acomete articulações que suportam o peso como os joelhos e os quadris. Sua prevalência aumenta muito com a idade, sendo observadas alterações radiográficas da OA em até $85 \%$ das pessoas com mais de 75 anos (SANTOS et al., 20Ira)

Frequentemente estão associados alguns fatores como a sobrecarga na articulação, excesso de peso, traumatismos (grandes, pequenos ou repetidos), como os que resultam de atividades desportivas ou profissionais, bem como outras doenças reumáticas ou malformações que danificam as articulações progressivamente (BASILIO, 2013).

Por sua vez, a osteoartrose tem uma etiologia multifatorial, mas as mudanças corporais associadas com o envelhecimento e obesidade são fatores fortemente associados à sua etiologia (AGUIAR, et al., 2015). O aumento da idade, o fator genético e a presença de outras doenças que afetam as estruturas articulares, influenciam a biomecânica das articulações e condicionam a um aumento do quadro inflamatório articular (DUARTE et al., 2013). 
É o tipo mais comum de artrite e afeta mais da metade das pessoas acima de 65 anos e, é mais prevalente em mulheres (MUSUMECI et al., 2015). Na osteoartrose, quer primária ou secundária, a cartilagem é o tecido que mais sofre com as alterações, a qual perde sua natureza homogênea, é rompida e fragmentada, com fibrilação, fissuras e ulcerações. Com o avanço da patologia, a cartilagem passa a não existir e áreas de osso subcondral ficam expostas (HUSSAIN et al., 2016).

A degradação da cartilagem articular ocorre de maneira lenta e progressiva, ocasionada pela insuficiência do condrócito em manter o equilíbrio entre renovação e degradação tecidual, prevalecendo à degradação. Com isso ocorrem fissuras na cartilagem articular e em sua estrutura, beneficiando a perda funcional da articulação, reduzindo a capacidade de absorção do impacto e da mobilidade articular podendo apresentar os seguintes sintomas, dor articular, rigidez matinal, crepitação, entre outros sintomas (UTH, TROFONOV, 2014).

\section{ANATOMIA E BIOMECÂNICA DO JOELHO}

Segundo Gozzi (2016), o joelho é o maior complexo articular do nosso corpo. Ele é formado por 4 ossos e 3 articulações. Esta articulação é a segunda que mais sofre lesão no nosso corpo, perdendo apenas para a articulação do ombro. Os 4 ossos que formam a articulação do joelho são: o fêmur, a tíbia, a patela e a fíbula. Esses ossos se articulam formando 3 articulações: femorotibial (entre o fêmur e a tíbia), patelofemoral (entre a patela e o fêmur), e a tíbiofibular proximal (entre a tíbia e a fíbula).

Essa articulação é classificada estruturalmente como sendo do tipo sinovial e funcionalmente como uma diartrose, é característica única de uma articulação sinovial a presença de um compartimento denominadode cavidade articular, esse espaço permite que a articulação possa realizar umavasta amplitude de movimento (GUPTON et al., 2020).

O joelho apresenta ligamentos que tem por finalidade sustentar, reforçar e estabilizar a articulação, entre eles o ligamento cruzado anterior, cruzadoposterior e os colaterais tibial e fibular. A cartilagem articular é um tecido que recobre a superfície articular, responsável por evitar o atrito entre as estruturas ósseas, porém apresenta pouca capacidade de reparo, pois é uma estrutura avascular (KATAGIRI et al., 2017). 
O músculo quadríceps femoral é um músculo localizado na região anteriorda coxa e apresenta importante repercussão na estabilidade do joelho, além disso, é um músculo que apresenta função de realizar a extensão do joelho (BORDONI; VARACALLO, 2020).

Realçando o conceito das articulações, estas fazem parte do grupo de componentes do corpo humano que o permitem mover e deslocar; umas têm como função formar uma camada protetora dos tecidos moles que revestem o esqueleto; outras têm como função ajudar aos movimentos de flexão, extensão ou mesmo adução e abdução; por último, existem articulações designadas móveis quem permitem uma variedade extensa de movimentos destacando-se a articulação do joelho (SILVA, 2015).

\section{PRINCIPAIS ALTERAÇÕES FUNCIONAIS}

À medida que muitos indivíduos vão envelhecendo, perdas funcionais podem ocorrer resultado da falta de atividade do sistema neuromuscular aliado a falta de força muscular e do condicionamento físico. Além desses fatores, alguns indivíduos em idade avançada podem desenvolver a retenção de líquido o que promove a redução de algumas substâncias necessárias para o bom condicionamento do corpo, contribuindo assim para o desenvolvimento da osteoartrite (GOMES-NETO et al., 2016).

Segundo Vieira et al. (2016), durante o envelhecimento percebemos limitação da flexibilidade e perda da força muscular, a limitação da flexibilidade está relacionada com a perda da força muscular, presença de encurtamentos musculotendíneos, diminuição da elasticidade da pele e alterações morfológicas periarticulares que predispõem o aparecimento de doenças osteoarticulares.

Dentre os aspectos mais importante presente na qualidade vida para os idosos, a capacidade funcional é o aspecto mais essencial considerado por eles, pois esse se baseia no princípio de realizar atividades, ir ao parque, as compras, viajar, entre outros tipos de lazeres que se caracterizam em ter uma vida independente (AMORIM, 2016).

Para Santos et al. (20irb), os indivíduos com oesteoartrite de joelho apresentam dificuldades em realizar atividades funcionais, principalmente aquelas que envolvem mobilidade e transferências. As principais alterações musculoesqueléticas envolvidas na 
osteoartrite de joelho são a dor, a rigidez articular e diminuição da força muscular dos músculos que compõe o quadríceps e os isquiostibiais, deixando o indivíduo susceptível a quedas quando o declínio funcional é importante. Com a redução da força flexora e extensora, a articulação do joelho fica exposta a sobrecargas mecânicas, que acentuam a concentração de forças na articulação do joelho, acentuando o desgaste da cartilagem articular.

A artrose de joelho leva a alterações funcionais objetivas, sendo a dificuldade mais comum subir e descer escada e a diminuição da velocidade da marcha. A intensidade da dor e um fator determinante para o relato das dificuldades, sendo o que mais interfere na velocidade usual e rápida da marcha, além de prejudicar os graus de amplitude muscular do movimento deste individuo (CECHETTI et al., 2012). Com o aumento progressivo da patologia, diversas alterações sensitivas e motoras, proprioceptivas ocorrem, entre elas o equilíbrio e o controle muscular diminuem ocasionando aumento da dor (GOMIERO et al., 2018).

Clinicamente, o principal sintoma da patologia é a dor localizada de caráter contínuo, a mesma é acentuada pela carga e pelos movimentos. A dor na maioria das vezes ocorre por irritação sinovial secundária, motivada pela destruição da cartilagem (SILVA; GAMA, 2017).

\section{FISIOTERAPIA AQUÁTICA E OS BENEFÍCIOS DA ÁGUA}

O meio aquático permite mobilização ativa precoce e o fortalecimento dinâmico, sendo a hidroterapia frequentemente prescrita nos casos de $\mathrm{AO}$ dos membros inferiores por serem articulações de sobrecarga . A utilização da água para fins terapêuticos possui vários sinônimos: hidrologia, hidrática, hidroterapia, hidroginástica, terapia pela água e exercícios na água. $\mathrm{Na}$ atualidade, o termo mais utilizado é reabilitação aquática ou hidroterapia. Considerado um recurso fisioterapêutico importante, a Hidroterapia utiliza piscinas aquecidas para a reabilitação de várias patologias, além de outras modalidades de terapia com a água. Esse conjunto de técnicas aquáticas, quando utilizadas por fisioterapeutas, costuma ser chamada de fisioterapia aquática (CASTRO et al., 2017).

A fisioterapia por meio do emprego de exercícios terapêuticos e utilizando os 
princípios físicos da água e seus efeitos fisiológicos, visa proporcionar a cura e a prevenção de doenças, além de ser considerada uma intervenção não-farmacológica e não-invasiva, que envolve diversas técnicas, podendo ser empregada conforme as necessidades de cada indivíduo a ela submetido. Praticada em piscinas aquecidas para tratamento de várias patologias ou disfunções com métodos específicos, utiliza as propriedades físicas da água como uma importante ferramenta, que fornece um ambiente ideal para indivíduos portadores de limitações na terapia em solo (FORNAZARI, 2012).

As propriedades terapêuticas da água permitem a prática de exercícios de maior amplitude de movimento e menores impactos, dificilmente realizados em solo, e por isso é uma das intervenções terapêuticas principais no tratamento da osteoartrite de joelho. $\mathrm{O}$ processo de recuperação funcional torna-se acelerado devido o aumento da mobilidade articular, do controle muscular, da resistência e alívio do quadro álgico (ANTUNES et al., 2020).

Os efeitos observados através do calor da água em que o paciente está em imersão vão ajudar a reduzir a dor, o espasmo muscular e induzir o relaxamento e uma maior amplitude de movimento (VIEIRA et al., 2016).

A fisioterapia aquática pode ser segura e eficaz no tratamento da OA do joelho por ocorrer submersão do membro inferior em água aquecida. A temperatura da água aumenta o limiar de dor, diminuindo o quadro álgico e dessa forma pode melhorar a capacidade funcional e a qualidade de vida. Esses são benefícios a curto prazo (ALCALDE et al., 2017).

Os efeitos fisiológicos da imersão estão associados aos seus princípios físicos, como a densidade, pois todo objeto ou corpo que colocado no meio aquático, que apresentar densidade menor do que a da água flutuará. Sendo a flutuação, ou empuxo, uma força com direção oposta à da gravidade, na água ela ocasiona uma menor descarga de peso corporal. Essa característica da água é fundamental para a hidroterapia, pois a densidade alterada permite uma redução considerável na pressão sobre suas articulações, permitindo exercícios mais completos e que atinjam uma melhor eficácia, reduzindo o risco de agravamento clínico (ROCHA et al., 2019). 
As propriedades físicas, fisiológicas e o aquecimento da água desempenham um papel importante na analgesia, na redução da rigidez e na redução do impacto articular, favorecendo a realização de exercícios de fortalecimento e alongamento muscular e exercícios de equilíbrio, pois não existem pontos de apoio, sendo o paciente obrigado a promover alterações posturais (SOUZA et al., 2017).

O calor da água diminui a sensibilidade das fibras nervosas rápidas (tato) e lentas (dor), liberando endorfinas que diminuem a sensação de dor, promovendo relaxamento muscular e, consequentemente, melhorando a mobilidade articular (BARDUZZI et al., 2013).

O empuxo da água resulta em descompressão das articulações, diminuindo o atrito articular, e faz com que os indivíduos se sintam mais leves, sendo esta uma forma segura de realizar exercícios aeróbios (ANTUNES et al., 2020).

Os princípios que afetam o processo terapêutico de reabilitação em meio líquido derivam de quase todos os princípios físicos que governam os comportamentos da água. Densidade, incompressibilidade e capacidade de reter e transmitir o calor da água que são forças intensas a confrontar. As conseqüências biológicas dessas forças podem afetar beneficamente quase todos os sistemas homeostáticos, esses efeitos fisiológicos surgem imediatamente após imersão, a transferência do calor começa e, sendo o calor específico do corpo humano diferente do da água, o corpo perde ou ganha calor mais rapidamente do que a água (SILVA; LIMA, 20II).

Em um estudo que avaliou o efeito da hidroterapia na força muscular e flexibilidade de idosos, foi constatado grandes feitos benéficos desse método para os idosos. Tendo isso em vista, o tratamento hidroterapêutico é de grande importância para manutenção da postura e equilíbrio físico e dinâmico em idosos da comunidade (MEEREIS et al., 2013).

Pela imersão do corpo, algumas das propriedades da água, entre elas a pressão hidrostática, conseguem potenciar a ativação cortical, especialmente das áreas sensoriais e motoras, o que se revela importante no processo de envelhecimento. Assim, a prática regular de atividades aquáticas permite a ocorrência deste mecanismo (SATO et al., 2012; SCHAEFER ET AL., 2016). 


\section{BENEFÍCIOS DA FISIOTERAPIA AQUÁTICA NA OSTEOARTROSE}

De acordo com Masselli et al. (2012), a redução da dor é um dos maiores benefícios trazidos pela hidroterapia ao portador de osteoartrose, além de ter boa aceitação dos exercícios pelo paciente, de poder reduzir a carga corporal, melhora a função física e a capacidade aeróbica. Estes benefícios são visíveis mesmo em diferentes graus da patologia.

Sekome et al. (2019) verificou em seus estudos que a modalidade terapêutica provocou redução do nível de dor dos pacientes, pois os benefícios que o meio aquático proporciona são associados às propriedades da água e temperatura adequada, a manutenção do calor na água durante a terapia ameniza a sensibilidade da fibra nervosa com rapidez e a exposição prolongada diminui a dor através da sensibilidade da fibra nervosa lenta.

Miotto et al. (2013), menciona a fisioterapia aquática como uma das principais intervenções terapêuticas no tratamento da osteoartrose, uma vez que as propriedades físicas e fisiológicas da água nos permite a realização de exercícios dificilmente executados em solo, essa vantagem associados à maior amplitude de movimento também permitido pela água e a temperatura elevada da mesma, aumentam a mobilidade articular, a resistência muscular, trazendo alívio de dores acelerando assim o processo de recuperação funcional. Wibelinger e Borges (2012), também apresenta a hidroterapia como uma modalidade muito eficiente no tratamento de osteoartrose, aonde seus benefícios vão além de físicos, traz benefícios também emocionais e sociais, confirma ainda que o uso da piscina aquecida mais a associação das propriedades físicas da água trazem ganhos significativos ao paciente.

Os exercícios terapêuticos aquáticos destacam-se como uma das principais intervenções fisioterapêuticas no tratamento da OA, pois devido as propriedades da água facilitam a execução dos exercícios reduzindo o impacto articular na região afetada, tornando o exercício menos doloroso. O calor da água diminui a sensibilidade ao tato e a dor, liberando endorfinas que diminuem a dor, promovem um relaxamento muscular e, consequentemente, melhora a mobilidade articular dos pacientes (SANTOS et al., 20Ira; BARDUZZI et al., 2013).

Outro efeito benéfico que a hidroterapia trás para os pacientes com osteoartrose de joelho é a melhora na velocidade e qualidade da deambulação, pois os exercícios aplicados 
em piscina terapêutica proporcionam uma melhora na reabilitação e devolvem a função aos pacientes acometidos proporcionando assim à base principal para uma independência funcional (MASIERO et al., 2018).

Há métodos que podem ser utilizados na terapia, tais como o Watsu, trata-se de uma técnica baseada através de movimentos passivos juntamente com a sustentação na água e o movimento contínuo rítmico da água, com isso ocorre um relaxamento profundo. O Bad ragaz é uma técnica que associa a flutuação do paciente com auxílio de flutuadores no pescoço, braços, pelve e pernas e a realização de exercícios funcionais baseado na técnica de facilitação neuromuscular proprioceptiva (FNP) oriunda do Kabat. O Halliwick utiliza atividades com o objetivo de facilitar padrões de movimento variando sua dificuldade, com isso ela foi baseada nos princípios de hidrodinâmica e no desenvolvimento humano, fazendo com que o paciente se torne mais independente na água (VERENGUE et al., 2021).

As intervenções de exercícios aquáticos foram todas estruturadas de forma diferente, e foram descritos como exercícios focados em força, resistência, equilíbrio e alongamento. Exercícios aeróbicos aquáticos, incluindo saltos, corrida, chutes, torções, polichinelos e degraus laterais (KIM et al., 2012; ANSARI et al., 2014). É uma combinação de peso corporal aqua-fitness, aqua-jogging com peso corporal, hidroterapia, aqua-fitness resistido ao aqua-jogging (FISKEN et al., 2015).

Belmonte et al. (2017), em seus estudos afirmaram que apesar de os exercícios aquáticos não modificarem a deterioração da cartilagem, o calor da água auxilia no relaxamento da musculatura estabilizadora da articulação, além da diminuição da tensão sobre a mesma pela flutuação, promovendo alívio da dor e auxiliando no processo de desaceleração da doença.

A tendência da hidroterapia é resultar na reabilitação e tratamento de pacientes com osteoartrose no joelho, proporcionando melhoras físicas e fisiológicas. $\mathrm{O}$ meio aquático facilita o alongamento, a força e agrega na amplitude articular do paciente. Tendo uma facilidade na execução dos exercícios, que são dificilmente executados em solo e que acaba gerando uma sobrecarga maior na articulação (KRUGER et al., 202I). 


\section{CONSIDERAÇÕES FINAIS}

A osteoartrose é uma das doenças que mais acomete a população mundial, principalmente os idosos, caracterizada pela perda progressiva da cartilagem articular, e a redução da lubrificação do líquido sinovial. E devido ao aumento da expectativa de vida e com o envelhecimento fisiológico, as doenças crônicas degenerativas vêm se tornando frequente, além de que para o idoso, leva a uma redução da qualidade de vida e capacidade funcional.

A atuação da fisioterapia, é de extrema importância para a população idosa, tanto na forma de tratamento, quanto na prevenção de doenças articulares como a osteoartrose, que pode evitar o agravamento de perda funcional da articulação.

Sendo assim, a fisioterapia aquática mostrou ser eficaz no tratamento e na redução do quadro álgico dos pacientes, pode- se ver que a água com seus princípios físicos auxilia no alívio de tensões reduz a ansiedade o que eleva a sensação de independência dentro da água, e com isso a renovação da autoestima. Assim associado os efeitos fisiológicos da água com movimentos e exercícios, o processo de reabilitação através da fisioterapia aquática é potencializado.

\section{REFERÊNCIAS}

I. ALCALDE, G.E.; FONSECA, A.C.; BÔSCOA, T.F.; GONÇALVES, M.R.; BERNARDO, G.C.; PIANNA, B.; CARNAVALE, B.F.; GIMENES, C.; BARRILE, S.R.; ARCA, E.A. Effect of aquatic physical therapy on pain perception, functional capacity and quality of life in older people with knee osteoarthritis: study protocol for a randomized controlled trial. Trials. v. I8, n. I, p. 317, 2017.

2. AMORIM, D.N.P. Associação da capacidade funcional com o perfil epidemiológico de idosos longevos. Dissertação (Pós-Graduação em Gerontologia). Universidade Católica de Brasília, 2016.

3. ANSARI, S.; ELMIEH, A.; HOJJATI, Z. Effects of aquatic exercise training on pain, symptoms, motor performance, and quality of life of older with knee osteoarthritis. Anals of Applied Sport Science. v. 2, n. 2, p. 29-38, 2014.

4. ANTUNES, B.T.; SILVEIRA, V.M.T.; BARBOSA, V.T.A.; SANTANA, J.E.; GOMES, A.S.M.V.; CARVALHO, P.C.V.; BARROS, N.L.M.; UCHÔA, L.B.P.E. Avaliação da marcha e do equilíbrio de pacientes idosos com osteoartrose de joelho. Brazilian Journal of Development. v. 6, n. 9, p. 72788-80o, 2020. 
5. BARDUZZI, G.O.; ROCHA-JÚNIOR, P.R.; SOUZA-NETO, J.C.; AVEIRO, M.C. Capacidade funcional de idosos com osteoartrite submetidos a fisioterapia aquática e terrestre. Fisioterapia em Movimento. v. 26, n. 2, p. 349-36o, 2013.

6. BASILIO, S. Intervenção da fisioterapia em indivíduos com osteoartrose do joelho: características da prática clínica, resultados obtidos e fatores de prognóstico para os resultados de sucesso. 217f. Dissertação (Mestrado em Fisioterapia em Condições MúsculoEsqueléticas). Universidade de Nova Lisboa, 2013.

7. BELMONTE, L.M.; GERENT, P.M.; SILVA, F.Z.; LIMA, I.A.X.; BELMONTE, L.A.O. Efeito do exercício aquático terapêutico em mulheres com osteoartrose de joelho: um estudo randomizado controlado. Fisisenectus. v. 5, n. I, p. 31-4I, 2017.

8. BORDONI, B.; VARACALLO, M. A. Anatomy, bony pelvis and lower limb, thigh quadriceps muscle. StatPearls, 2020.

9. CASTRO, F.F.; CASTRO, L.C.C.; CARVALHO, L.O.; SBARDELOTT, Y.A.S.; SOUZA, J.N.; MARTINELLI, P.M. Hidroterapia no tratamento da osteoartrite de quadril: revisão bibliográfica. Revista de Ciência em Foco. v. I, n. I, p. 64-72, 2017.

Io. CECHETTI, F.; FABRO, Q.A.; MARTINI, R.D. Reabilitação aquática como recurso de tratamento de osteoartrose de quadril e joelho. Fisioterapia Brasil. v. 13, n. 5, p. 384-389, 2012.

II. DUARTE, V.S.; SANTOS, M.L.; RODRIGUES, K.A.; RAMIRES, J.B.; AREAA, G.P.T.; BORGE, G.F. Exercícios físicos e osteoartrose: uma revisão sistemática. Fisioterapia em Movimento. v. 26, n. I, p. 193-202, 2013.

I2. FISKEN, A.L.; WALTERS, D.L.; HING, W.A.; STEELE, M.; KEOGH, J.W. Comparative effects of 2 aqua exercise programs on physical function, balance, and perceived quality of life in older adults with osteoarthritis. Journal of Geriatric Physical Therapy. v. 38, n. I, p. 17-27, 2015.

13. FORNAZARI, P.L. Fisioterapia aquática, 2012. Disponível em: http://repositorio.unicentro.br:8o8o/jspui/handle/123456789/503. Acesso em 23 de abril de 2021.

14. GOZZI, R. Articulação do Joelho. 20r6. Disponível em: http://anatomiafacil.com.br/oo6-articulacao-do-joelho. Acesso em 24 abril de 202I.

I5. GOMES-NETO, M.; ARAÚJO, A.D.; JUNQUEIRA, I.D.A.; OLIVEIRA, D.; BRASILEIRO, A.; ARCANJO, F.L. Estudo comparativo da capacidade funcional e qualidade de vida entre idosos com osteoartrite de joelho obesos e não obesos. Revista Brasileira de Reumatologia. v. 56, n. 2, p. 126-130, 2016.

16. GOMIERO, A.B.; KAYO, A.; ABRAÃO, M.; PECCIN, M.S.; GRANDE, A.J.; TREVISANI, V.F. Sensory-motor training versus resistance training among patients 
with knee osteoarthritis: randomized single-blind controlled trial. São Paulo Medical Journal. v. 136, n. I, p. 44-50, 2018.

17. GUPTON, M.; IMONUGO, O.; TERREBERRY, R.R. Anatomy, bony pelvis and lower limb. StatPearls, 2020.

18. HUSSAIN, S.M.; NEILLY, D.W.; BALIGA, S.; PATIL, S.; MEEK, R.M.D. Knee osteoarthritis: a review of management options. Scottish Medical Journal. v. 6I, n. I, p. 7I6, 2016.

19. KATAGIRI, H.; MENDES, L.F.; LUYTEN, F.P. Definition of a critical size osteochondral knee defect and its negative effect on the surrounding articular cartilage in the rat. Osteoarthritis and Cartilage, v. 25, n. 9, p. 1531-1540, 2017.

2o. KAWANO, M.M.; ARAÚJO, A.L.I.; CASTRO, C.M.; MATOS, A.M. Avaliação da qualidade de vida em pacientes portadores de osteoartrose de joelho. Acta Ortopédica Brasileira. v. 23, n. 6, p. 307-310, 2015.

2I. KIM, I.S.; CHUNG, S.H.; PARK, Y.J.; KANG, H.Y. The effectiveness of an aquarobic exercise program for patients with osteoarthritis. Applied Nursing Research. v. 25, n. 3, p. I8I-I89, 2012.

22. KRUGER, C.R.P.; SILVA, I.M.; SAMPAIO, A.C.R.I. A eficácia da hidroterapia em pacientes com osteoartrose de joelho. Revista de Iniciação Científica e Extensão. v. 4, n. I, 595-601, 2021.

23. MASSELli, M.R.; MORITA, A.K.; PACHIONI, C.A.S.; FERREIRA, D.M.A. Efeitos dos exercícios aquáticos na osteoartrite de quadril ou joelho: revisão. Colloquium Vitae. v. 4, n. I, p. 53-61, 2012.

24. MASIERO, S.; VITTADINI, F.; FERRONI, C.; BOSCO, A.; SERRA, R.; FRIGO, A.C.; FRIZZIERO, A. The role of thermal balneotherapy in the treatment of obese patient with knee osteoarthritis. International Journal of Biometeorology. v. 62, n. 2, p. 243-252, 2018.

25. MEEREIS, E.C.W.; FAVRETTO, C.; SOUZA, J.; GONÇALVES, M.P.; MOTA, C.B. Influência da hidrocinesioterapia no equilíbrio postural de idosas institucionalizadas. Motriz: Revista de Educação Física. v. 19, n. 2, p. 269-277, 2013.

26. MIOTTO, C.; KAYSER, B.; MOLIN, D.V.; KUMER, A.J.; WIBELINGER, M.L. Tratamento fisioterapêutico das artralgias. Revista Dor. v. I4, n. 3, p. 216-218, 2013.

27. MUSUMECI, G.; AIEllO, F.C.; SZYCHLINSKA, M.A.; DI ROSA, M.; CASTROGIOVANNI, P.; MOBASHERI, A. Osteoarthritis in the XXIst century: risk factors and behaviours that influence disease onset and progression. International Journal of Molecular Sciences. v. 16, n. 3, p. 6093-6112, 2015.

28. OLIVEIRA, T.V.C.; CARVALHO, R.R.J.; CÂNDIDO, E.A.F.; LIMA, P.A.L.; SANTANA, L.S. Avaliação da efetividade da cirurgia de artroplastia total de joelho 
associada a fisioterapia sob o ponto de vista da funcionalidade. ScireSalutis. v. 3, n. 2, p. 6172, 2013.

29. ROCHA, D.H.; SANTOS, S.Y.; MORAIS, G.S.; PEREIRA, B.G.R.; SALEME, F.P.A. Hidroterapia como recurso terapêutico em idosos com diagnóstico de osteoartrose de joelho. Revista Saúde dos Vales. v. I, n. I, p. 155-167, 2019.

30. SANTOS, C.F.; SOUZA, R.M.P.; NETO, T.J.; ATALLAH, N.G. Tratamento da dor associada à osteoartrose de joelho em idosos: um ensaio clínico aleatório e duplamente encoberto com o clonixinato de lisina. Revista Dor. v. I2, n. I, p. 6-I4, 20112.

3i. SANTOS, M.L.A.D.S.; GOMES, W.F.; QUEIROZ, B.Z.; ROSA, N.M.D.B.; PEREIRA, D.S.; DIAS, J.M.D.; PEREIRA, L.S.M. Desempenho muscular, dor, rigidez e funcionalidade de idosas com osteoartrite de joelho. Acta Ortopédica Brasileira. v. I9, n. 4, p. 193-197, 2011b.

32. SANTOS, D.A.T.; AMARAL, A.F. Correlação entre flexibilidade de cadeia muscular posterior com qualidade de vida na osteoartrose de joelho. ıop. Monografia (Graduação em Fisioterapia). Centro Universitário UniGuairacá, 2020.

33. SATO, D.; ONISHI, H.; YAMASHIRO, K.; IWABE, T.; SHIMOYAMA, Y.; MARUYAMA, A. Water immersion to the femur level affects cerebral cortical activity in humans: functional near-infrared spectroscopy study. Brain Topography. v. 25, n. 2, p. 220$227,2012$.

34. SCHAEFER, S.Y.; LOUDER, T.J.; FOSTER, S.; BRESSEL, E. Effect of water immersion on dual-task performance: implications for aquatic therapy. Physiotherapy Research International. v. 21, n. 3, p. 147-154, 2016.

35. SEKOME, K.; MADDOCKS, S. The short-term effects of hydrotherapy on pain and self-perceived functional status in individuals living with osteoarthritis of the knee joint. The South African Journal of Physiotherapy. v. 75, n. I, 2019.

36. SILVA, J.M.C. Modelo biomecânico do joelho humano. Dissertação (Mestrado Integrado em Engenharia Mecânica). Faculdade de Engenharia da Universidade do Porto, 2015 .

37. SILVA, O.N.A.; GAMA, L.W.C. Hidroterapia como método de tratamento da osteoartrose do joelho: revisão de literatura. 22p. Monografia (Graduação em Fisioterapia). Centro Universitário São Lucas, 2017.

38. SILVA, S.D.A.; LIMA, P.L. Os benefícios da reabilitação aquática para grupos especiais. EFDeportes.com. Ano 16, n. 16o, 2011.

39. SOUZA, A.A.; MOURA, A.J.; BASTONE, C.A. Efetividade de um programa de fisioterapia aquática na capacidade aeróbia, dor, rigidez, equilíbrio e função física de idosos com osteoartrite de joelho. Fisioterapia Brasil. v. I8, n. 2, p. 165-171, 2017. 
40. UTH, C.; TROFONOV, D. Stem cell application for osteoarthritis in the knee joint: a minireview. World Journal Stem Cells. v. 6, n. 5, p. 629-636, 2014.

4I. VERENGUE, B.R.; CALININ, G.; ALMEIDA, G.R.; ALVES, J.L.; PRAÇA, M.L.; RAPOSO, N.; RODRIGUES, R.G. Proposta de protocolo para reabilitação de osteoartrose de joelho em cinesioterapia e hidroterapia e protocolo de reabilitação para osteoartrose. Revista Inova Saúde. v. II, n. 2, 202I.

42. VIEIRA, R.J.; ALVES, O.M.; LUZES, R. Efeitos da hidroterapia em pacientes idosos com osteoartrose de joelho. Revista Discente da UNIABEU. v. 4, n. 8, p. II-I5, 2016.

43. WIBELINGER, L.M.; BORGES, A.M. Hidroterapia em portador de artrite reumatoide. Revista Brasileira de Ciência das Saúde. ano Io, v. 31, p. 6I-66, 2012. 